\title{
MMP-9 Contributes to Dendritic Spine Remodeling Following Traumatic Brain Injury
}

\author{
Barbara Pijet (D, Marzena Stefaniuk, and Leszek Kaczmarek $(D$ \\ Laboratory of Neurobiology, BRAINCITY, Nencki Institute of Experimental Biology of Polish Academy of Sciences, Pasteura 3, \\ 02-093 Warsaw, Poland
}

Correspondence should be addressed to Barbara Pijet; b.pijet@nencki.gov.pl and Leszek Kaczmarek; 1.kaczmarek@nencki.gov.pl

Received 23 November 2018; Accepted 3 April 2019; Published 6 May 2019

Guest Editor: Michael Nilsson

Copyright (C) 2019 Barbara Pijet et al. This is an open access article distributed under the Creative Commons Attribution License, which permits unrestricted use, distribution, and reproduction in any medium, provided the original work is properly cited.

\begin{abstract}
Traumatic brain injury (TBI) occurs when a blow to the head causes brain damage. Apart from physical trauma, it causes a wide range of cognitive, behavioral, and emotional deficits including impairments in learning and memory. On neuronal level, TBI may lead to circuitry remodeling and in effect imbalance between excitatory and inhibitory neurotransmissions. Such change in brain homeostasis may often lead to brain disorders. The basic units of neuronal connectivity are dendritic spines that are tiny protrusions forming synapses between two cells in a network. Spines are dynamic structures that undergo morphological transformation throughout life. Their shape is strictly related to an on/off state of synapse and the strength of synaptic transmission. Matrix metalloproteinase-9 (MMP-9) is an extrasynaptically operating enzyme that plays a role in spine remodeling and has been reported to be activated upon TBI. The aim of the present study was to evaluate the influence of MMP-9 on dendritic spine density and morphology following controlled cortical impact (CCI) as animal model of TBI. We examined spine density and dendritic spine shape in the cerebral cortex and the hippocampus. CCI caused a marked decrease in spine density as well as spine shrinkage in the cerebral cortex ipsilateral to the injury, when compared to sham animals and contralateral side both 1 day and 1 week after the insult. Decreased spine density was also observed in the dentate gyrus of the hippocampus; however, in contrast to the cerebral cortex, spines in the DG became more filopodia-like. In mice lacking MMP9 , no effects of TBI on spine density and morphology were observed.
\end{abstract}

\section{Introduction}

Traumatic brain injury caused by an external mechanical force evokes a variety of brain responses, including focal extrasynaptic matrix degradation, neuronal loss within hippocampus area, glia activation, synaptic remodeling, and ion channels activity changes $[1,2]$. On a neurotransmission level, a massive glutamate efflux, increased level of extracellular glutamate, and hyperactivation of NMDAR receptor channels followed by their loss are observed [3]. These events are also strictly related to dendritic spine remodeling [4]. Dendritic spines are small membranous protrusions that undergo plastic morphological changes under both physiological (e.g., development or learning and memory) and pathological (e.g., neurodegeneration, psychiatric disorders) conditions [5-7]. Several recent reports have described changes in dendritic spine density and size following brain trauma [8-16].
MMP-9 is pericellularly acting endopeptidase, classified as a gelatinase due to its ability to cleave gelatin [17-21]. Through involvement in extracellular matrix remodeling, it regulates numerous cell processes and physiological functions [22-26]. Aside from physiological role, MMP-9 takes part in such central nervous system pathological events as injury, stroke, or epileptogenesis, as well as neuropsychiatric disorders such as schizophrenia or addiction [27-35]. Importantly, previous reports have also indicated that MMP-9 is a crucial dendritic spine shape modulator [36-41] and its level is altered posttrauma [28, 40, 42-45]. To what extent upon brain injury MMP-9 is involved in altering dendritic spines number and shape is yet unknown. To bridge this gap, we set out to analyze the effects of MMP-9 levels on TBIstimulated plastic changes of the dendritic spines in the mouse brain. For this, we used controlled cortical impact (CCI) as an animal model of traumatic brain injury [46]. 
First, we describe influence of CCI on density and morphology of dendritic spines in the cerebral cortex and hippocampus 24 hours and 7 days of postbrain injury. Next, we assess the effects of missing MMP-9 due to the gene knockout (KO) on spine density and shape following TBI.

\section{Materials and Methods}

2.1. Animals. The study was performed on adult (12-14 weeks old) C57BL/6J male mice (Animal House, Center for Experimental Medicine, Białystok, Poland), MMP-9 homozygous knockout mice (MMP-9 KO), and their WT siblings (MMP-9 WT) on a C57BL/6J background [47]. All mice were maintained in the Animal House of the Nencki Institute. Animals were housed in individual cages under controlled environment (temperature $22 \pm 1^{\circ} \mathrm{C}$, humidity $50-60 \%$, with free access to food and water and a $12 \mathrm{~h}$ light/dark cycle). All procedures were performed in accordance with the Animal Protection Act in Poland, directive 2010/63/EU, and were approved by the 1st Local Ethics Committee (Permissions Numbers: 383/2012; 609/2014).

2.2. Induction of TBI with CCI. Mice were subjected to unilateral cortical contusion using the controlled cortical impact protocol $[28,46,48]$ following anesthesia evoked with $4 \%$ isoflurane (Aerrane; Baxter, UK) in $100 \%$ oxygen with a delivery rate of $4 \mathrm{l} / \mathrm{min}$. During the surgery, concentration of isoflurane was maintained at the level of 3\% in 100\% oxygen with delivery of $0.61 / \mathrm{min}$ (Combi Vet Anesthesia System; Rothacher; Switzerland). For deeper sedation, briefly before the injury, mice were injected subcutaneously with butorphanol (10 $\mu \mathrm{g} / 30 \mathrm{~g}$ body weight). After skull exposure by midline scalp incision, craniectomy was performed using a $5 \mathrm{~mm} \varnothing$ trephine (Fine Science Tools FST; Germany) over the left parietotemporal cortex between the lambda and bregma (Figure 1(a)). The bone piece was carefully removed without disrupting the dura. For TBI execution, we used Leica Impact One device equipped with an electrically driven metallic piston controlled by a linear velocity displacement transducer (Leica Biosystems, KAWA.SKA; Poland). After craniectomy, the adjustable CCI equipment was mounted on the left stereotaxic arm at an angle of $20^{\circ}$ from vertical. CCI was delivered according to the protocol [28] using the following parameters: $\varnothing 3 \mathrm{~mm}$ : flat tip; depth: $0.5 \mathrm{~mm}$ from the dura; velocity: $5 \mathrm{~m} / \mathrm{s}$, and dwell time: $100 \mathrm{~ms}$. After injury, bleeding was strictly controlled, a piece of sterile plastic was placed over the craniectomy area, and the incision was sutured with nylon stitches (Sigmed; Poland). Next, the animals were returned to the heated home cages for postsurgical recovery. Sham-injured animals underwent identical anesthesia and craniectomy procedures, but were not subjected to CCI. The following number of animals was subjected to procedure per each time point: C57BL/6J ( $n=5 \mathrm{CCI}, n=5$ sham), MMP-9 WT ( $n=5$ CCI, $n=3$ sham), and MMP-9 KO ( $n=5$ CCI, $n=3$ sham $)$.

2.3. Nissl Staining. To verify cerebral cortex degeneration, we performed Nissl staining. 24 hours and 7 days after the injury, mice were anesthetized and perfused with $0.37 \%$ sulfide solution $\left(5 \mathrm{ml} / \mathrm{min}, 4^{\circ} \mathrm{C}\right)$ for $5 \mathrm{~min}$ followed by perfusion with $4 \%$ paraformaldehyde in $0.1 \mathrm{M}$ sodium phosphate buffer, $\mathrm{pH} 7.4\left(5 \mathrm{ml} / \mathrm{min}, 4^{\circ} \mathrm{C}\right)$ for $10 \mathrm{~min}$. The brains were removed from the skull and postfixed in buffered $4 \%$ paraformaldehyde for $4 \mathrm{~h}$ at $4^{\circ} \mathrm{C}$, and then cryoprotected in a solution containing $30 \%$ glycerol in $0.02 \mathrm{M}$ potassium phosphate-buffered saline for $48 \mathrm{~h}$. Samples were then frozen on dry ice and stored at $-80^{\circ} \mathrm{C}$. Frozen brains were sectioned in the coronal plane $(40 \mu \mathrm{m})$ with a sliding cryostat (Leica Biosystems, KAWA.SKA; Poland). The sections were mounted on microscope gelatin-covered slides, dried, and stained with cresyl violet. Pictures of Nissl-stained sections were taken using the light microscope Nikon Eclipse $\mathrm{Ni}$ equipped with PlanApo $2 \mathrm{x}$ objective.

2.4. Dendritic Spine Analysis. Dendritic spines were visualized using lipophilic dye Dil $\left(1,1^{\prime}\right.$-dioctadecyl-3,3,3',3'-tetramethylindocarbocyanine perchlorate, \#D282 Life Technologies, Warsaw, Poland). 24 hours and 7 days after CCI, mice were sacrificed and their brains were collected. Next, they were cut into $130 \mu \mathrm{m}$ sections on vibratome (Leica VT 1000S, Leica Biosystems Nussloch GmbH, Wetzlar, Germany). Slices were processed for Dil staining. Random dendrite labeling was performed using $1.6 \mu \mathrm{m}$ tungsten particles (Bio-Rad, Hercules, CA, USA) coated with Dil. Dye was delivered to cells using Gene Gun (Bio-Rad). After staining, slices were fixed with $0.4 \%$ paraformaldehyde in phosphate-buffered saline (PBS; overnight at $4^{\circ} \mathrm{C}$ ) and placed on microscopic slides. $Z$-stacks of dendrites from the 2 nd and 3 rd layers of the perilesional cortex and the dentate gyrus (DG) were acquired using the LSM780 confocal system equipped with 40x objective (Plan Apochromat 40x/1.4 Oil DIC) (Zeiss, Poznań, Poland). Dil emission was excited using a HeNe $594 \mathrm{~nm}$ laser. For each image, the following parameters were applied: $70 \mathrm{~nm}$ pixel size, $300 \mathrm{~nm} Z$-intervals, averaging 4. Maximum intensity projections of $Z$-stacks covering the length of dendrite were analyzed using semiautomatic SpineMagick! software [49]. It allows marking dendritic spine head and base manually. Next, the software marks automatically spine edges that can be adjusted manually to fully reflect the spine shape [49]. For each animal, 5-7 single dendrites from selected brain areas (one dendrite per neuron per image) were analyzed. First, dendritic spine density was calculated. In the next step, dendritic spines were examined according to the following morphological parameters: spine area, head width, spine length, and a scale-free parameter - the length divided by the width (length to width ratio) (Figure 2(a)). This parameter reflects the spine shape-the higher the ratio, the more filopodial spine is.

To determine group size, we used assumption on the basis of sample size determination and the following equation:

$$
n=1+2 C\left(\frac{s}{d}\right)^{2}
$$

where $n$ is the group size, $C$ is a constant dependent on the value of $\alpha$ and power selected (here it equals 10.51 for 0.9 power and 0.05 significance level), $s$ is an estimate of the 

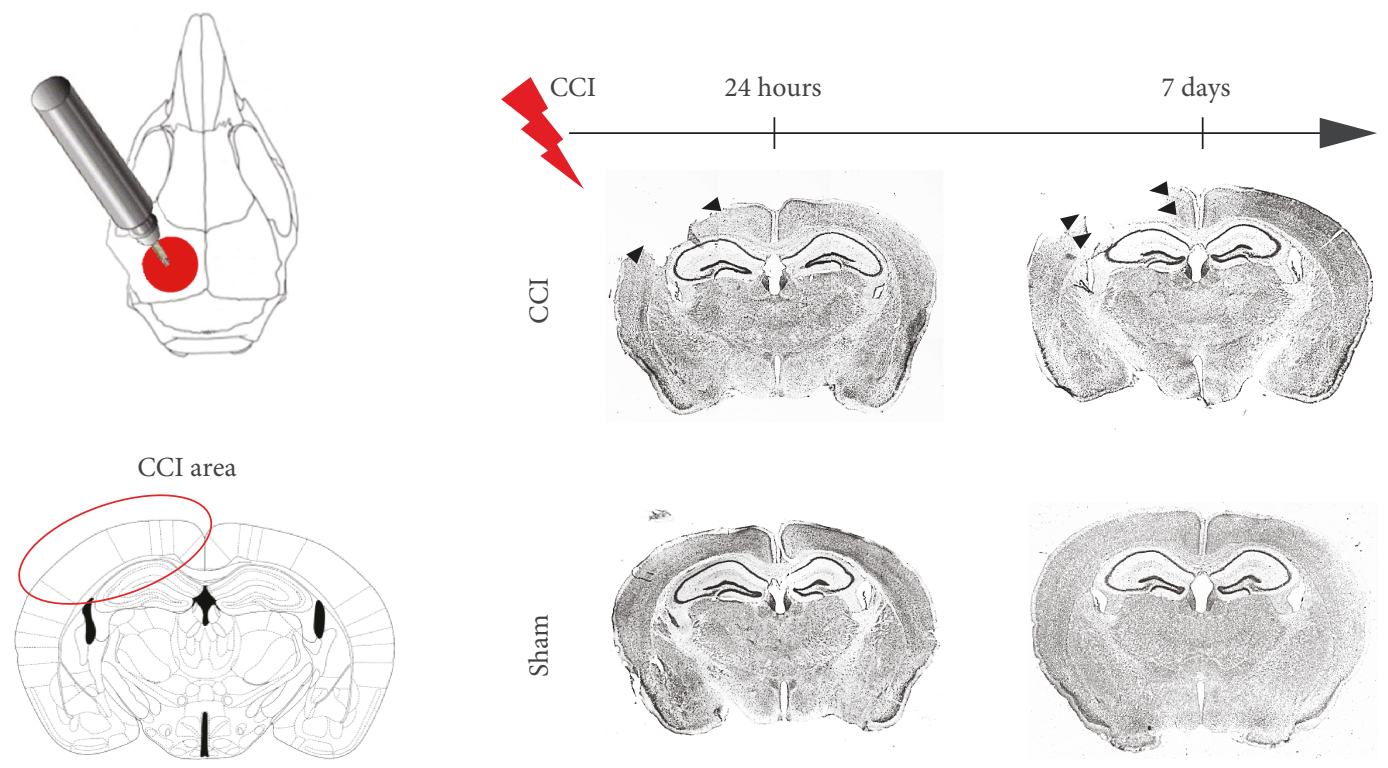

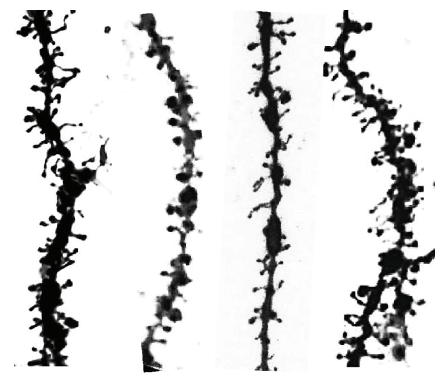

Sham (7 d) Ipsi (24 h) Ipsi (7 d) Contra (7 d)

Cortex
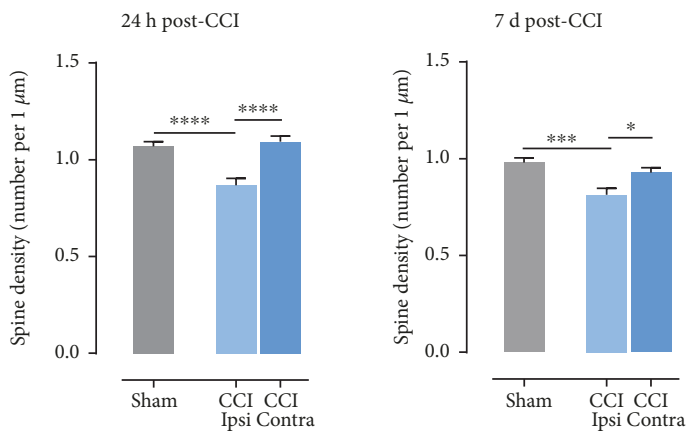

(b)

(a)

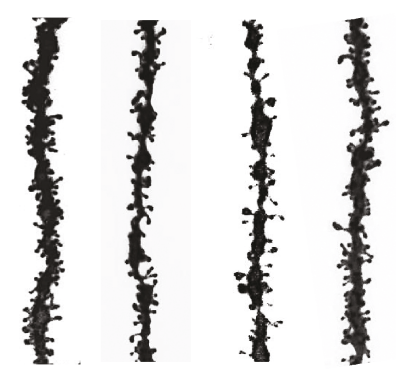

Sham (7 d) Ipsi (24 h) Ipsi (7 d) Contra (7 d)

Hippocampus (DG)

$24 \mathrm{~h}$ post-CCI

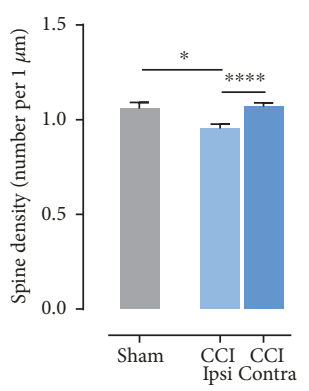

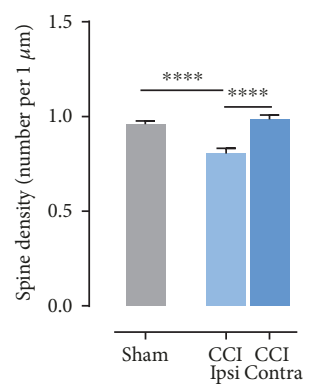

(c)

Figure 1: Decrease in spine density after controlled cortical impact (CCI). (a) Schematic representation of injured area. Nissl-stained brain sections from animals at 1 and 7 days after CCI (CCI: animals after controlled cortical impact; sham: animals subjected to craniectomy without cortical injury). (b) Spine density (number of spines per $1 \mu \mathrm{m}$ of dendrite length) in ipsi- and contralateral $2 \mathrm{nd}$ and $3 \mathrm{rd}$ cortex layers of C57Bl6/J mice, 1 and 7 days after CCI and sham procedures; right panel shows representative dendrites pictures. (c) Spine density in ipsi- and contralateral dentate gyrus of C57Bl6/J mice, 1 and 7 days after CCI and sham procedures; right panel shows representative dendrite pictures. Data are presented as mean \pm SEM. Statistical analysis was carried out using one-way ANOVA followed by Tukey's post hoc test. Asterisks indicate statistical significance from the CCI and sham groups, respectively. ${ }^{*} P<0.05 ;{ }^{* * *} P \leq 0.001$; ${ }^{* * * *} P<0.0001$.

population standard deviation of the variable, and $d$ is the magnitude of the difference. With the above-estimated values, the group size is 10 . In our experiments, we increased the group size and it varied between 15 and 25 depending on the experiment (5 to 7 pictures per animal, 3-5 animals per group). 


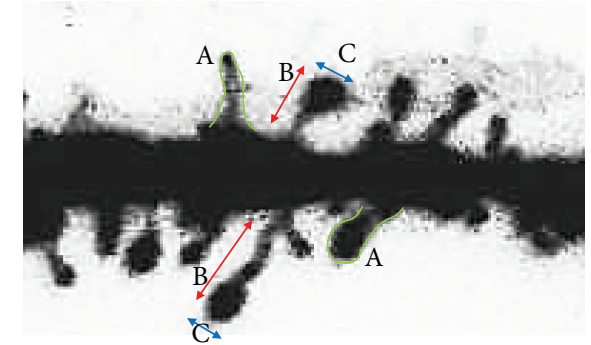

Dendritic spine

shape parameters

$$
\begin{array}{lll}
\mathrm{A} & : & \text { spine area } \\
\mathrm{B} & : & \text { spine length } \\
\mathrm{C} & : & \text { head width } \\
\mathrm{B} / \mathrm{C} & : & \text { length/width ratio }
\end{array}
$$

(a)

Cortex

$24 \mathrm{~h}$ post-CCI

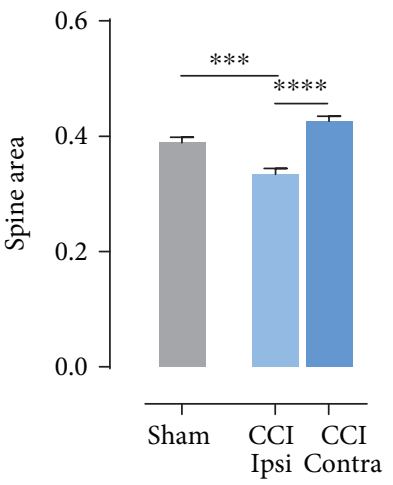

Cortex
$7 \mathrm{~d}$ post-CCI

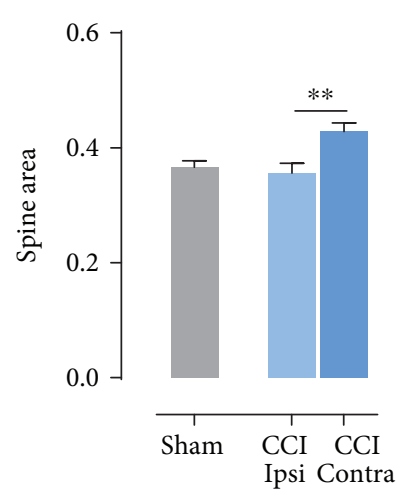

Hippocampus (DG)

$24 \mathrm{~h}$ post-CCI

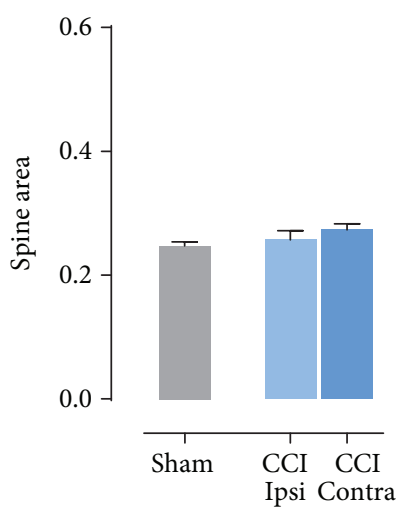

$7 \mathrm{~d}$ post-CCI

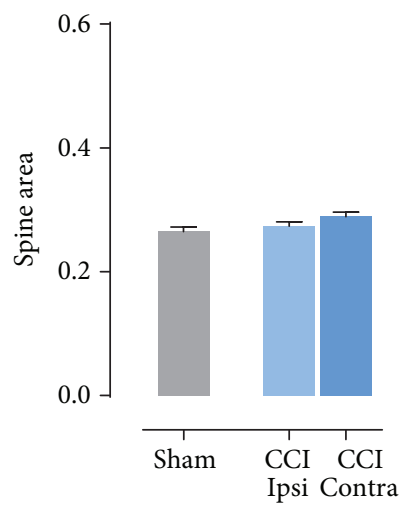

(b)

Hippocampus (DG)

$24 \mathrm{~h}$ post-CCI

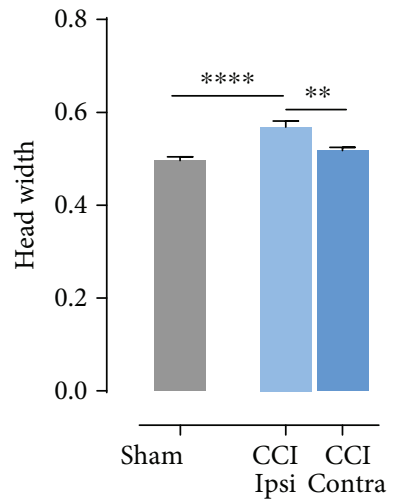

$7 \mathrm{~d}$ post-CCI

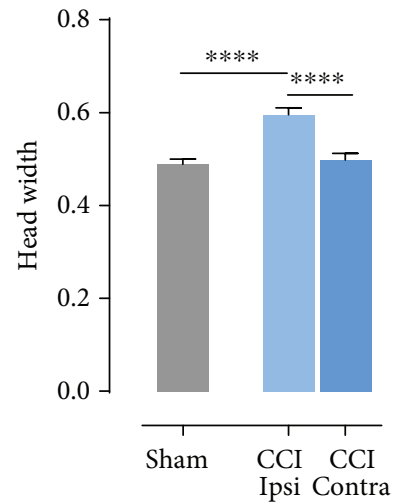

$24 \mathrm{~h}$ post-CCI

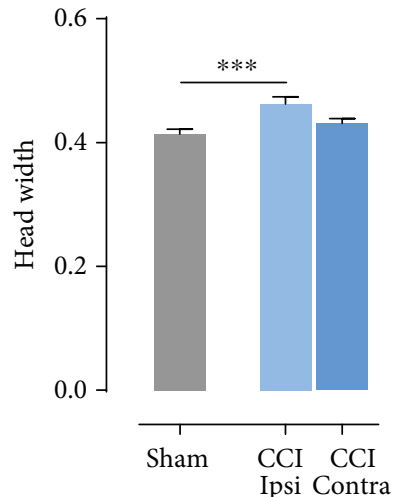

$7 \mathrm{~d}$ post-CCI

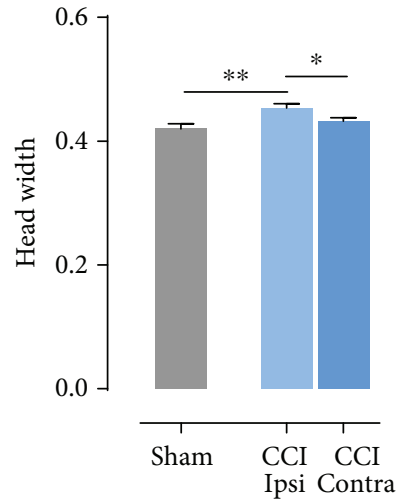

(c)

Figure 2: Continued. 
Cortex

$24 \mathrm{~h}$ post-CCI

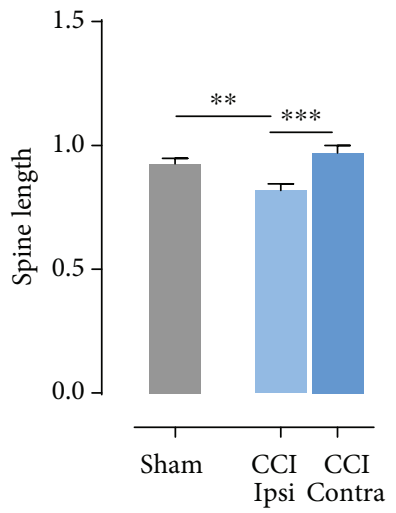

Cortex

$24 \mathrm{~h}$ post-CCI

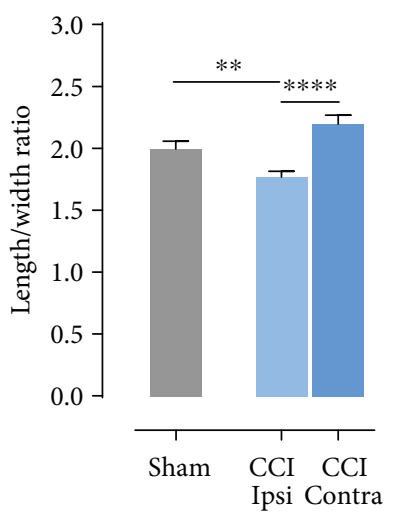

7 d post-CCI

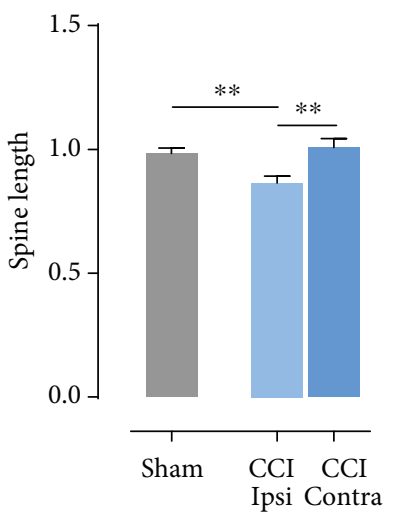

(d)

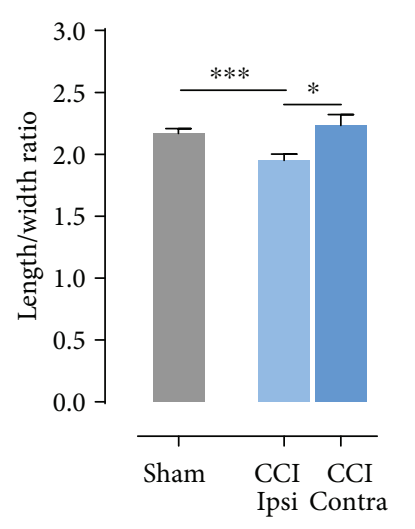

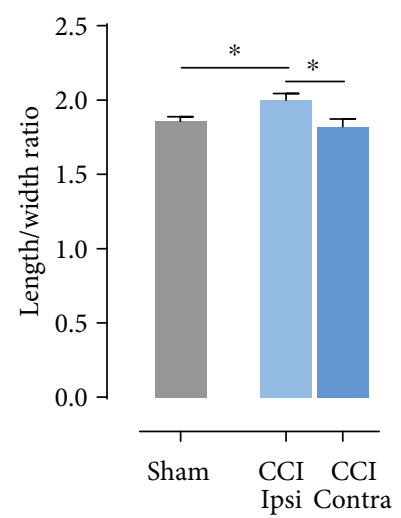

Hippocampus (DG)
$24 \mathrm{~h}$ post-CCI

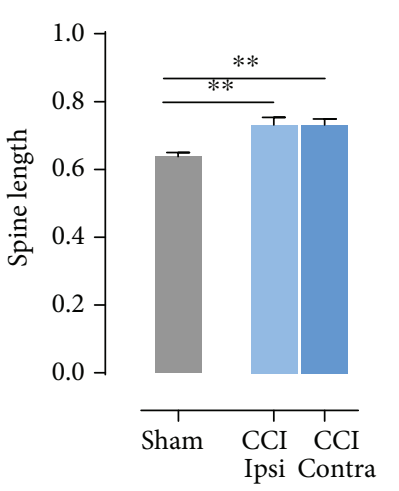

7 d post-CCI

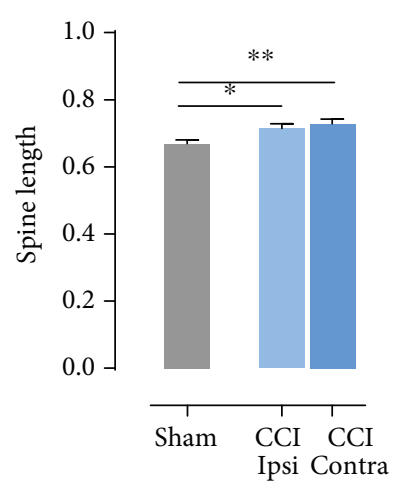

(e)

$$
24 \mathrm{~h} \text { post-CCI } 7 \mathrm{~d} \text { post-CCI }
$$

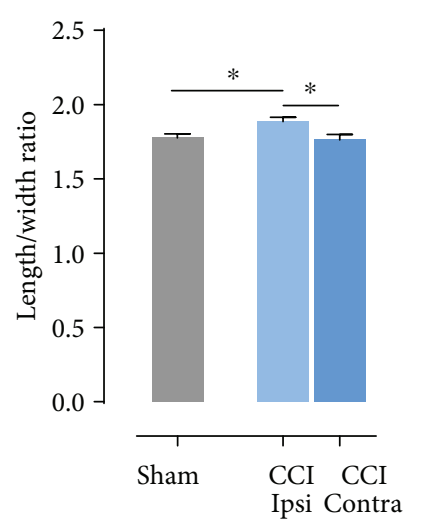

FIGURE 2: Time-dependent changes in dendritic spines shape after controlled cortical impact (CCI). (a) Spine shape parameters: A: spine area; B: spine length; C: head width; B/C: length/width ratio. (b) Spine area calculated in the ipsi- and contralateral cortex and hippocampus of C57Bl6/J mice, 1 and 7 days after CCI and sham procedures. (c) Head width calculated in the ipsi- and contralateral cortex and hippocampus of C57B16/J mice, 1 and 7 days after CCI and sham procedures. (d) Spine length calculated in the ipsi- and contralateral cortex and hippocampus of C57B16/J mice, 1 and 7 days after CCI and sham procedures. (e) Length/width ratio calculated in the ipsi- and contralateral cortex and hippocampus of C57Bl6/J mice, 1 and 7 days after CCI and sham-operated animals. Data are presented as mean \pm SEM. Statistical analysis was carried out using one-way ANOVA followed by Tukey's post hoc test. Asterisks indicate statistical significance from the CCI and sham groups, respectively. ${ }^{*} P<0.05 ;{ }^{* *} P \leq 0.01 ;{ }^{* * *} P \leq 0.001 ;{ }^{* * * *} P<0.00013 .3$.

2.5. Statistical Analyses. All results are expressed as mean \pm SEM. The appropriate tests were chosen (see below), taking into account whether data had normal distribution and equal variation. All analyses were conducted using GraphPad Prism, version 7.02 (GraphPad Software Inc., La Jolla, CA). Differences between the experimental groups were considered significant if the type 1 error was less than $5 \%$.

\section{Results}

3.1. Decrease in Spine Density in the Cerebral Cortex and the Hippocampus Evoked by Controlled Cortical Impact (CCI). To induce TBI in mice, we used controlled cortical impact
(CCI) as described by Bolkvadze and Pitkänen [46]. To describe morphological changes evoked by injury, the brains were collected 24 hours and 7 days post-CCI. Control animals (sham-operated) were subjected to craniectomy only and sacrificed together with the animals that underwent CCI. The Nissl staining revealed time-dependent cerebral cortex degeneration within the injured area (Figure 1(a)). In sham-operated animals, hardly any tissue damage was observed. A separate batch of animals was used to perform dendritic spine analyses. Animals were subjected to CCI or sham surgery. Twenty-four hours or 7 days later, their brains were collected to perform analyses of dendritic spines from ipsi- and contralateral sides. For this, we used lipophilic dye that incorporates into cell membranes and marks the whole 
cell contour. Sections were imaged using a confocal microscope, as described in Materials and Methods. Spine density was calculated as a number of protrusions per $1 \mu \mathrm{m}$ of dendrite length. Twenty-four hours and 7 days after CCI, spine density was decreased in the 2 nd and 3rd layers of the ipsilateral cerebral cortex, as compared to the contralateral hemisphere $\left(24 \mathrm{~h}{ }^{* * * *} P<0.0001 ; 7 \mathrm{~d} * P=0.0135\right)$ and sham animals $\left(24 \mathrm{~h}{ }^{* * * *} P<0.0001 ; 7 \mathrm{~d} \quad{ }^{* * *} P=0.0003\right.$; Figure 2(b)). Similar effect was observed in the DG where 24 hours and 7 days after TBI spine density were significantly lower compared to the contralateral side $\left(24 \mathrm{~h}{ }^{*} \mathrm{P}<0.0001\right.$; $7 \mathrm{~d}{ }^{*} P=0.0001$; Figure $\left.1(\mathrm{c})\right)$ and sham-operated animals $\left(24 \mathrm{~h}^{*} P=0.0251 ; 7 \mathrm{~d}^{* * * *} P<0.0001\right.$; Figure $\left.1(\mathrm{c})\right)$.

3.2. Dendritic Spines Become Shorter and Wider in Injured Cerebral Cortex Area. Next, we aimed at more detailed analysis of morphological alterations following trauma. For this, we used a semiautomatic software to evaluate dendritic spine shapes on the basis of the following parameters: length, head width, and length to width ratio that describes the spine shape, with its increase reflecting filopodial shape (Figure 2(a)). The parameters were measured at two time points, 1 and 7 days after CCI. Dendritic spines shrank in the ipsilateral cerebral cortex following trauma as their areas were smaller both 24 hours and 7 days after brain injury, compared to the contralateral cortex $\left(24 \mathrm{~h}{ }^{* * * *} P<0.0001\right.$; $7 \mathrm{~d}{ }^{* *} P=0.0057$; Figure 1(b)). Significant difference between ipsilateral hemisphere and sham-operated animals was observed only after 24 hours $\left({ }^{* * *} P=0.0005\right)$, while in the DG, no significant differences were observed (Figure 2(b)). Head width (width at the widest point of the spine) increased both in the cortex and DG in injured hemispheres, compared to the contralateral hemisphere (cortex: $\left.24 \mathrm{~h}{ }^{* *} P<0.0015 ; 7 \mathrm{~d}{ }^{* * *} P>0.0001 ; \mathrm{DG}: 7 \mathrm{~d}{ }^{*} P=0.033\right)$ and animals that underwent sham surgeries (cortex: $24 \mathrm{~h}$ ${ }^{* * * *} P>0.0001 ; 7 \mathrm{~d}{ }^{* * *} P>0.0001$; DG: $24 \mathrm{~h}{ }^{* * *} P=0.0005$; $7 \mathrm{~d}^{* *} P=0.0086$ ) (Figure 2(c)). Next parameter, spine length, reflects the distance from the bottom to the top of the spine (Figure 2(d)). In the ipsilateral cortex, spine length was decreased compared to the contralateral hemisphere in both time points $\left(24 \mathrm{~h}{ }^{* * *} P=0.0006 ; 7 \mathrm{~d}{ }^{* *} P=0.0097\right)$ and shamoperated animals $\left(24 \mathrm{~h}{ }^{* * *} P=0.0021 ; 7 \mathrm{~d}{ }^{*} P=0.0043\right)$. In contrast, in the ipsilateral DG, spines were longer than in animals after sham operation $\left(24 \mathrm{~h}{ }^{* *} P=0.0021 ; 7 \mathrm{~d}\right.$ $\left.{ }^{*} P=0.0166\right)$, and no difference compared to the contralateral hemisphere was observed. Decrease of the ratio between the length and width of the spine was observed in the ipsilateral cerebral cortex when compared to the contralateral side (Figure 2(e), $24 \mathrm{~h}:{ }^{* *} P<0.0001 ; 7 \mathrm{~d}{ }^{*} P=0.0182$ ) and sham animals $\left(24 \mathrm{~h}:{ }^{* *} P=0.0061 ; 7 \mathrm{~d}{ }^{* * *} P=0.0010\right)$. While in the ipsilateral DG, opposite effect was noticed, where the length/width ratio increases in response to TBI compared to contralateral DG $\left(24 \mathrm{~h}{ }^{*} P=0.0166\right.$; $7 \mathrm{~d} * P=0.026)$ as well as in comparison to sham animals $\left(24 \mathrm{~h} * P=0.0402 ; 7 \mathrm{~d}{ }^{*} P=0.037\right.$; Figure $\left.2(\mathrm{e})\right)$.

3.3. Deficiency of MMP-9 Impairs the Effect of Brain Injury on Spine Density Decline. Since MMP-9 is one of the key modulators of dendritic spines shape and its role in TBI and subsequent epileptogenesis has been recently highlighted [28], we set out to evaluate whether the lack of MMP-9 affects morphological changes observed in WT animals. For this, we used mice missing MMP-9 (MMP-9 KO) and their wild-type littermates (WT MMP-9). We focused on 7-day post-CCI time point. As we reported in the previous report, MMP-9 activity was the highest during first week after brain injury [28]. After CCI in WT and KO MMP-9 animals, we analyzed dendritic spines as described above. 1 week after the brain injury, spine density was decreased in the ipsilateral cerebral cortex of WT animals as compared to the contralateral side of the injured brain $\left({ }^{* *} P=0.0011\right.$; Figure $\left.3(\mathrm{a})\right)$ and shamoperated animals $\left({ }^{* *} P=0.0067\right.$; Figure $\left.3(\mathrm{a})\right)$. However, this phenomenon was not observed in MMP-9 KO mice (Figures 3(a) and 3(b)).

3.4. MMP-9 Is Required for Spine Shape Remodeling upon Injury. To further unravel the influence of MMP-9 on the postinjury morphological changes of dendritic spines, we analyzed basic spine parameters (Figure 2(a)). We focused on length to width ratio and spine head width as these parameters indicate whether protrusions undergo plastic changes. In WT animals, in the ipsilateral cerebral cortex area, we observed increase in head width compared to contralateral hemisphere $\left({ }^{* * *} P<0.0001\right.$; Figure $\left.4(\mathrm{a})\right)$ and sham-operated animals $\left({ }^{*} P=0.0477\right.$; Figure $\left.4(\mathrm{a})\right)$. Similarly, to the cerebral cortex, in the ipsilateral dentate gyrus of hippocampus WT MMP-9 mice, head width was significantly bigger compared to the contralateral hippocampus $\left({ }^{* *} P=0.0020\right.$; Figure 5(a)) and sham-operated animals $\left({ }^{* *} \mathrm{P}=0.0061\right.$; Figure 5(a)). On the contrary, in MMP-9 KO animals, following CCI, no alterations in dendritic spine morphology were detected in both analyzed structures (Figures 4(a) and 5(a)). In the injured cerebral cortex, WT MMP-9 mice length/width ratio decreased, while in the ipsilateral dentate gyrus increased (Figures 4(b) and 5(b)). The changes in the ipsilateral cortex was significant compared to the contralateral side of the brain $\left({ }^{* *} P=0.0048\right.$; Figure 4(b)) and sham animals $\left({ }^{*} P=0.0316\right.$; Figure $\left.4(\mathrm{~b})\right)$, whereas in MMP-9 KO mice, no differences were observed (Figure $4(\mathrm{~b})$ ). In the DG, ratio between the spine length and width in ipsilateral hemisphere was significantly higher compared only to sham animals $\left({ }^{* *} P=0.0016\right.$; Figure $\left.5(\mathrm{~b})\right)$. Similarly, to the cortex, the changes between the CCI and sham groups were not significant (Figure 5(b)).

\section{Discussion}

Here, we show that traumatic brain injury caused by the controlled cortical impact induces acute (within 1 day post-TBI) changes in the dendritic spine number and morphology, as well as prolonged (up to 7 days after the injury) spine remodeling. Spine density decreases following brain trauma in the ipsilateral side both in the cerebral cortex and the dentate gyrus of the hippocampus. Following trauma dendritic spines in the ipsilateral cerebral cortex shrink, get shorter and their heads get wider, thus being converted into more mushroomlike shape. On the other hand, spines in the DG on the 


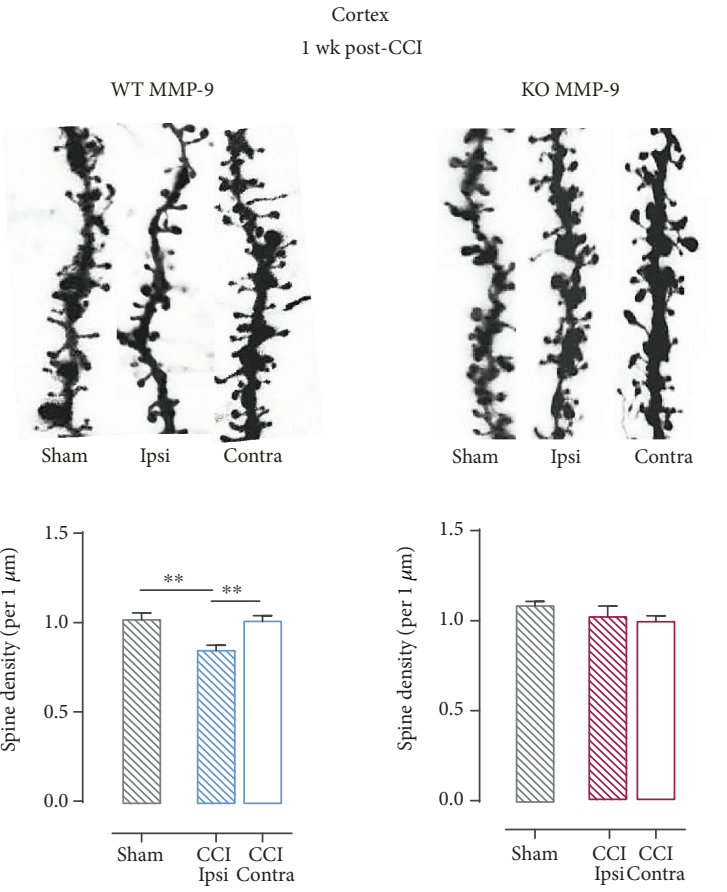

(a)

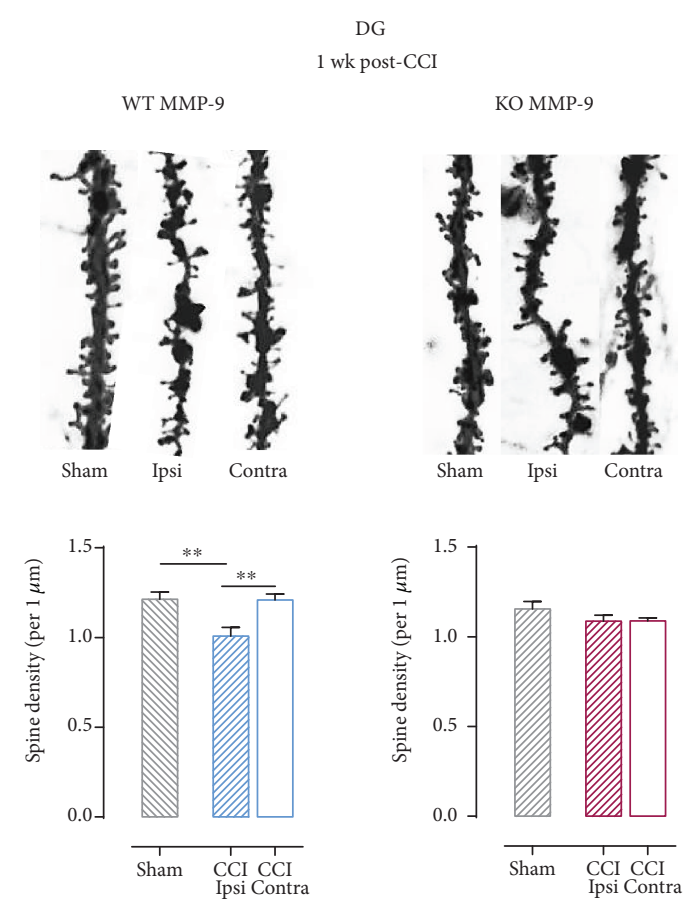

(b)

FIGURE 3: Effect of lack of functional MMP-9 on spine density in animals after controlled cortical impact (CCI). (a) Spine density in ipsi- and contralateral 2nd and 3rd cortex layers of animals with different $m m p-9$ gene expression levels 1 week after CCI and sham surgeries; upper panel shows representative dendrites pictures. (b) Spine density in the ipsi- and contralateral dentate gyrus of animals with different $m m p-9$ gene expression levels 1 week after CCI and sham surgeries; upper panel shows representative dendrite pictures. Data are presented as mean \pm SEM. Statistical analysis was carried out using one-way ANOVA followed by Tukey's post hoc test. Asterisks indicate statistical significance from the CCI and sham groups, respectively. ${ }^{* *} P \leq 0.01$.

ipsilateral side get longer and thinner, assuming more filopodial form. Lack of MMP-9 activity in the brain abrogates the effects evoked by the trauma, both as far as the spine dynamics (reflected by changes in the density) and morphological plasticity are concerned.

Dendritic spines are protrusions containing the majority of excitatory synapses, thus gating inputs received by the nerve cell $[4,50]$. The density and morphology of dendritic spines are regulated by synaptic activity, and so spines undergo dynamic turnover throughout life. Filopodiashaped spines are more prominent in the developing brain and are considered "immature" [51]. Some reports indicate that these are spine precursors during synapse formation [52]. Spines considered "mature" are more mushroomshaped, allowing stabilizing the spine by gathering more neurotransmitter receptors in the head [53]. Other feature of such shape is that the narrow spine neck might also compartmentalize calcium necessary for synaptic transmission to occur [54]. When the brain is challenged by injury, spines respond accordingly to maintain milieu homeostasis [7].

First, we measured dendritic spine density. Spine number has been shown to be related to overall synaptic activity, e.g., in the hippocampus transient enhancement of dendritic spine density accompanies early long-term potentiation (LTP) in the dentate gyrus [55]. Previous studies on dendritic spine plasticity evoked by TBI in animal models showed decreased spine density in the hippocampus and the cerebral cortex at various times after brain injury ( 24 hours to 35 days) $[9,11,13,16]$. Furthermore, one study expands these observations up to 1.5 years, showing changes that last not only shortly after brain injury but also continue into chronic stages of TBI. Moreover, they take place in widespread regions beyond the site of acute trauma [12]

Dendritic spine loss is accompanied by dendrite deformation and swelling [15]. Our results are essentially in agreement with the abovementioned data; however, they extend those observations by describing the detailed shape parameters. In the present study, we show that 24 -hour postinjury dendritic spine density decreases in the ipsilateral side in the cortex and the hippocampus, which is consistent with the previous report $[8,11]$. We further demonstrate that 7 days after injury the number of spines per dendrite length is still decreased in the cortex and the DG. Dendritic spine loss one-week posttrauma was also shown in the CA1 of the hippocampus [12]. Since our TBI conditions were far less severe, we conclude that even mild injury then can result in substantial changes in the brain.

Furthermore, we also show a detailed morphological analysis of dendritic spines in the cortex and hippocampus after traumatic brain injury. Here, we demonstrate that after brain trauma induced by CCI, dendritic spines in the cerebral cortex, ipsilaterally to the injury side, are getting shorter and their heads become wider, when compared to sham-operated animals and the contralateral side of the injured cerebral cortex. Overall, the spine plasticity observed in the cerebral cortex ipsilateral to the 
Cortex

WT MMP-9

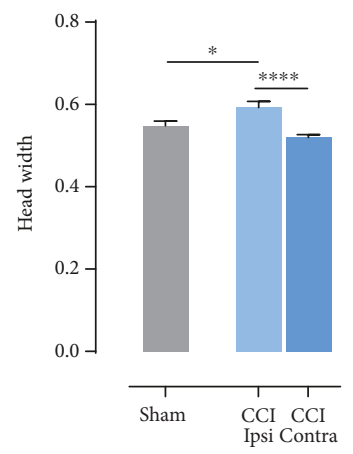

(a)

WT MMP-9

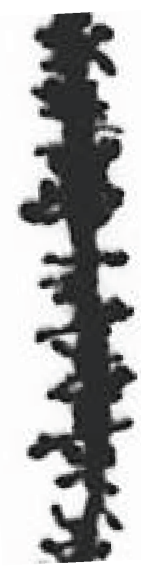

Sham

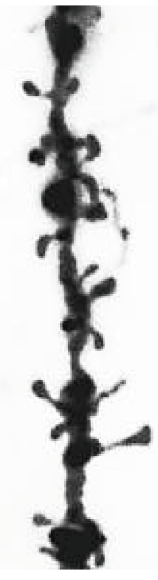

Ipsi

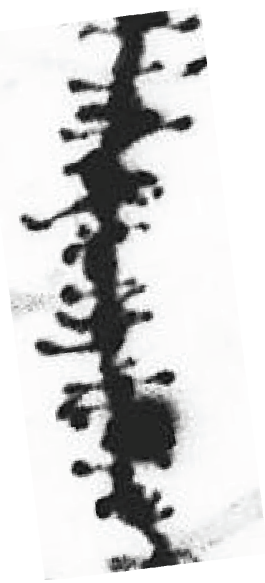

Contra (c)

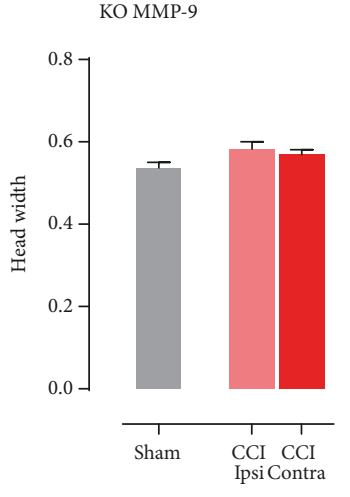

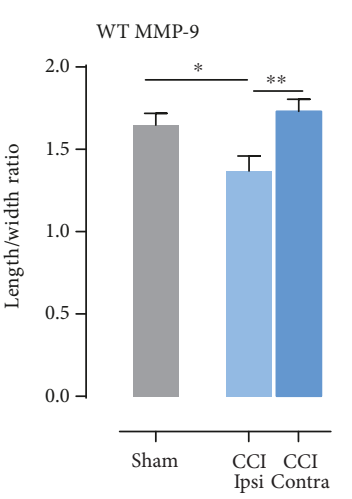

Cortex

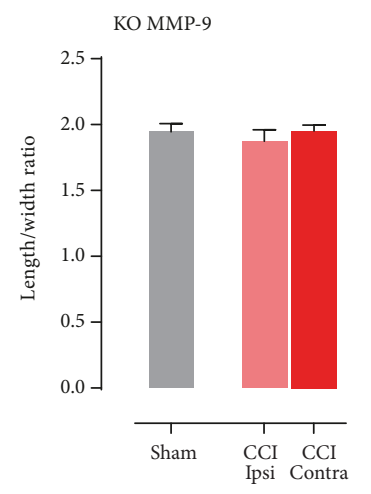

(b)

KO MMP-9

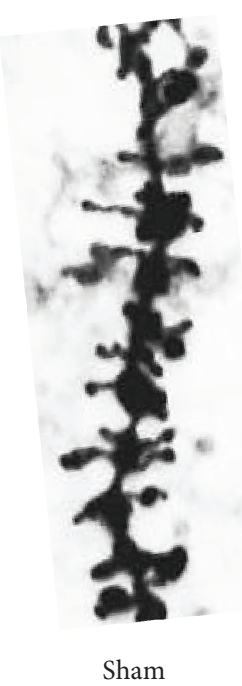

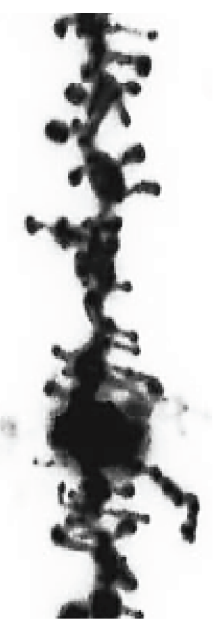

Ipsi

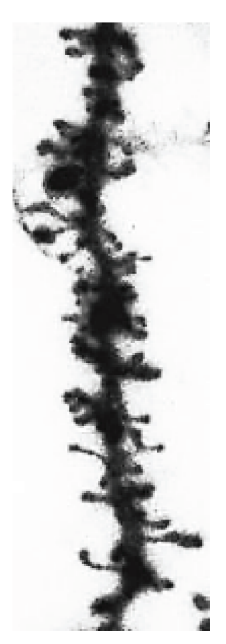

Contra (d)

FIGURE 4: Effects of MMP-9 on spine shape changes in the cerebral cortex layers of animals 1 week post-CCI. (a) Head width calculated in the ipsi- and contralateral cortex and hippocampus of C57Bl6/J mice, 7 days after CCI and sham procedures. (b) Length/width ratio calculated in the ipsi- and contralateral cortex and hippocampus of C57Bl6/J mice, 7 days after CCI and sham-operated animals. (c) Dendrite pictures from the ipsi- and contralateral cortex and sham animals. (d) Representative dendrite pictures from the ipsi- and contralateral cortex and sham animals. Data are presented as mean \pm SEM. Statistical analysis was carried out using one-way ANOVA followed by Tukey's post hoc test. Asterisks indicate statistical significance from the CCI and sham groups, respectively. ${ }^{*} P<0.05 ;{ }^{* *} P \leq 0.01$.

injury and in a deeper located DG showed similar pattern of spine morphological alterations, except for the length/width ratio. This morphological parameter is believed to reflect spine maturity $[4,39,52]$. The greater this value, the spine is more filopodial-shaped, i.e., immature. This finding deserves a special comment. The filopodia-like spines are presumably prone to support initiation of synaptic plasticity processes $[4,36,50]$. Therefore, this result may suggest that DG may undergo synaptic plasticity that may predispose this brain structure to support epileptogenesis that is a frequent consequence of TBI $[56,57]$.

In the present study, we show that TBI-driven dynamics and morphological plasticity of dendritic spines are MMP-9dependent. Increases in MMP-9 following TBI have previously been reported both in the animal brain and human cerebrospinal fluid, blood plasma, and serum $[9,30,44-47$,
58, 59]. The possible role of MMP-9 in controlling spine dynamics has been previously demonstrated in the DG, following treatment with excitotoxic kainic acid, where MMP$9 \mathrm{KO}$ mice were found to be resistant to spine loss [60, 61]. Similarly, recently, Nagaoka et al. [62] have reported that MMP-9 controls spine dynamics in the neocortex of fragile $\mathrm{X}$ mental retardation protein $\mathrm{KO}$ mice.

Furthermore, in our study, missing MMP-9 has entirely abrogated dendritic spine morphological plasticity provoked by TBI, both in the cerebral cortex ipsilateral to the injury and in the dentate gyrus. This finding goes well along with multiple data showing a pivotal role of MMP-9 in regulation of dendritic spine size and shape $[29,63,64]$.

Finally, we shall stress that previous studies $[28,40]$ demonstrated that MMP-9 KO mice brain showed limited brain damage after CCI. However, the extend of this 


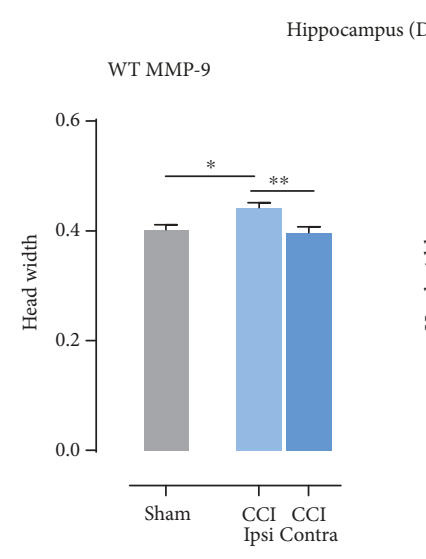

(a)

WT MMP-9

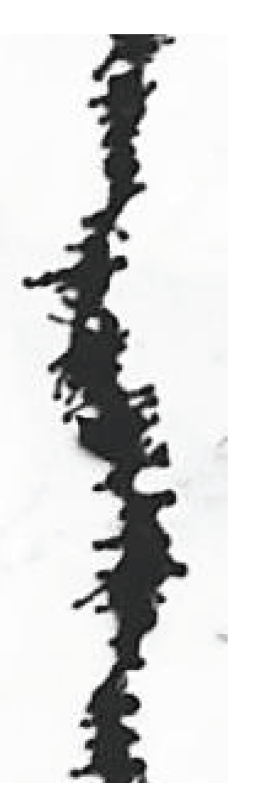

Sham

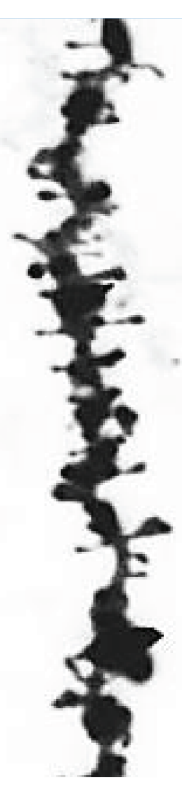

Ipsi
KO MMP-9

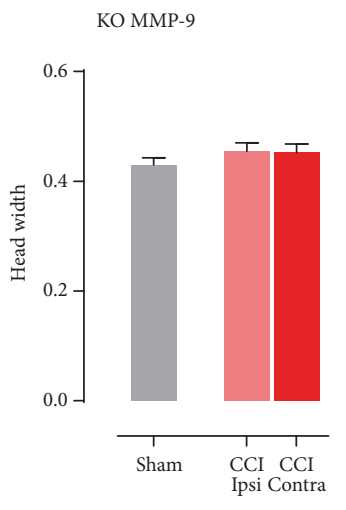

(a)

(c)

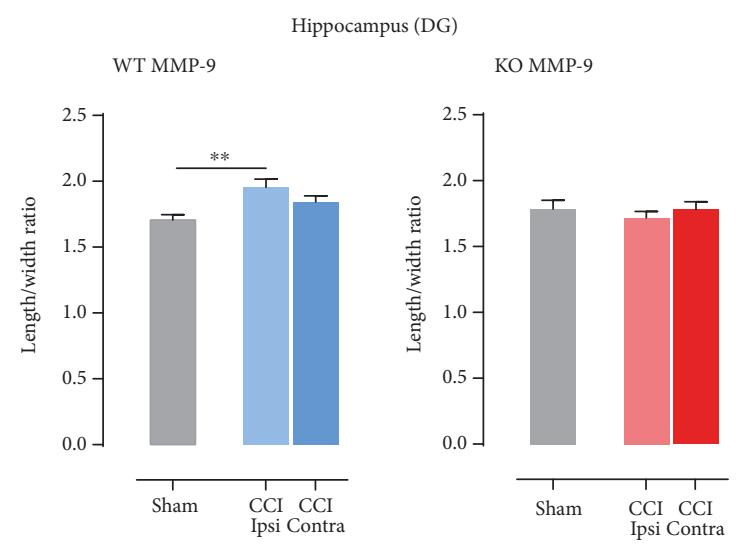

(b)

KO MMP-9

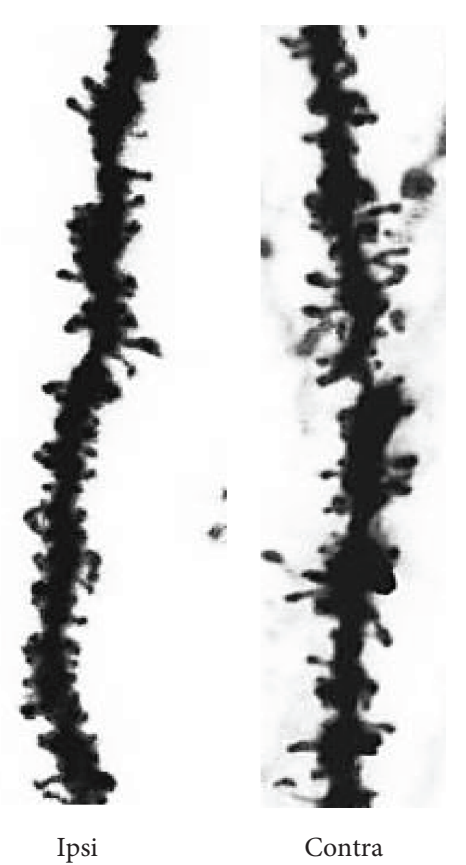

(d)

FIGURE 5: Effects of MMP-9 on spine shape changes in the dentate gyrus of animals 1 week post-CCI. (a) Head width calculated in the ipsiand contralateral hippocampus of C57Bl6/J mice, 7 days after CCI and sham procedures. (b) Length/width ratio calculated in the ipsi- and contralateral hippocampus of C57Bl6/J mice, 7 days after CCI and sham-operated animals. (c) Dendrite pictures from the ipsi- and contralateral dentate gyrus and sham animals. (d) Representative dendrite pictures from the ipsi- and contralateral dentate gyrus and sham animals. Data are presented as mean \pm SEM. Statistical analysis was carried out using one-way ANOVA followed by Tukey's post hoc test. Asterisk indicate statistical significance from the CCI and sham groups, respectively. ${ }^{*} P<0.05 ;{ }^{* *} P \leq 0.01$.

protection against brain damage (MMP-9 KO still demonstrated almost $40 \%$ of the cortical injury area as compared to WT) does not seem to explain the entire abrogation of TBI effects on the spines in the MMP-9 KO brains.

\section{Conclusions}

Herein, we have provided a detailed analysis of TBI-evoked density and morphological plasticity of the dendritic spines. We have found that in result of the injury, there is a decrease in spine density both very close (ipsilateral cerebral cortex) and more distal (hippocampal dentate gyrus on the ipsilateral side) to the locus of the injury. However, the spines located on neurons close to the injury assume more mushroom-like shape, whereas those in DG become more filopodia-like. Missing MMP-9 previously shown to exert control of the spine density and morphology abrogated the aforementioned plasticity entirely. Considering the previously reported role of MMP-9 in posttraumatic epileptogenesis (PTE) that might be supported by abnormal synaptic plasticity, and a welldocumented role of this enzyme in the plasticity of dendritic spines, it is tempting to suggest that MMP-9-dependent dendritic spine dynamics and morphological plasticity contribute to PTE. 


\section{Data Availability}

Previously reported (MMP-9 activity after CCI; role of MMP-9 in posttraumatic epileptogenesis) data were used to support this study and are available at doi:10.1007/s12035018-1061-5. These prior studies (and datasets) are cited at relevant places within the text as reference [28].

\section{Conflicts of Interest}

The authors declare that there is no competing interest relevant to the publication of this paper.

\section{Acknowledgments}

The study was supported by a grant from the National Center of Research and Development (NCBiR) grants: ERA-NET Neuron grant (NEURON/09/13) and (PBS/A8/36/2015) and the Foundation for Polish Science BRAINCITY MAB/ 2018/10. The BRAINCITY project is carried out within the International Research Agendas programme of the Foundation for Polish Science cofinanced by the European Union under the European Regional Development Fund.

\section{References}

[1] C. M. Atkins, "Decoding hippocampal signaling deficits after traumatic brain injury," Translational Stroke Research, vol. 2, no. 4, pp. 546-555, 2011.

[2] T. M. Reeves, M. L. Prins, J. P. Zhu, J. T. Povlishock, and L. L. Phillips, "Matrix metalloproteinase inhibition alters functional and structural correlates of deafferentation-induced sprouting in the dentate gyrus," The Journal of Neuroscience, vol. 23, no. 32, pp. 10182-10189, 2003.

[3] E. Shohami and A. Biegon, "Novel approach to the role of NMDA receptors in traumatic brain injury," CNS \& Neurological Disorders - Drug Targets, vol. 13, no. 4, pp. 567-573, 2014.

[4] C. Sala and M. Segal, "Dendritic spines: the locus of structural and functional plasticity," Physiological Reviews, vol. 94, no. 1, pp. 141-188, 2014.

[5] E. G. Gray, "Axo-somatic and axo-dendritic synapses of the cerebral cortex: an electron microscope study," Journal of Anatomy, vol. 93, pp. 420-433, 1959.

[6] K. M. Harris and S. B. Kater, "Dendritic spines: cellular specializations imparting both stability and flexibility to synaptic function," Annual Review of Neuroscience, vol. 17, no. 1, pp. 341-371, 1994.

[7] R. F. McCann and D. A. Ross, "A fragile balance: dendritic spines, learning, and memory," Biological Psychiatry, vol. 82, no. 2, pp. e11-e13, 2017.

[8] J. N. Campbell, D. Register, and S. B. Churn, "Traumatic brain injury causes an FK506-sensitive loss and an overgrowth of dendritic spines in rat forebrain," Journal of Neurotrauma, vol. 29, no. 2, pp. 201-217, 2012.

[9] X. Gao, P. Deng, Z. C. Xu, and J. Chen, "Moderate traumatic brain injury causes acute dendritic and synaptic degeneration in the hippocampal dentate gyrus," PLoS One, vol. 6, no. 9, article e24566, 2011.

[10] E. Schwarzbach, D. P. Bonislawski, G. Xiong, and A. S. Cohen, "Mechanisms underlying the inability to induce area CA1 LTP in the mouse after traumatic brain injury," Hippocampus, vol. 16, no. 6, pp. 541-550, 2006.

[11] C. N. Winston, D. Chellappa, T. Wilkins et al., "Controlled cortical impact results in an extensive loss of dendritic spines that is not mediated by injury-induced amyloid-beta accumulation," Journal of Neurotrauma, vol. 30, no. 23, pp. 19661972, 2013.

[12] A. Ertürk, S. Mentz, E. E. Stout et al., "Interfering with the chronic immune response rescues chronic degeneration after traumatic brain injury," The Journal of Neuroscience, vol. 36, no. 38, pp. 9962-9975, 2016.

[13] X. Gao and J. Chen, "Mild traumatic brain injury results in extensive neuronal degeneration in the cerebral cortex," Journal of Neuropathology \& Experimental Neurology, vol. 70, no. 3, pp. 183-191, 2011.

[14] Y.Zhang, Z. G. Zhang, M. Chopp et al., "Treatment of traumatic brain injury in rats with $\mathrm{N}$-acetyl-seryl-aspartyl-lysyl-proline," Journal of Neurosurgery, vol. 126, no. 3, pp. 782-795, 2017.

[15] S. Zhao, X. Gao, W. Dong, and J. Chen, "The role of 7,8-dihydroxyflavone in preventing dendrite degeneration in cortex after moderate traumatic brain injury," Molecular Neurobiology, vol. 53, no. 3, pp. 1884-1895, 2016.

[16] J. R. Chen, T. J. Wang, Y. J. Wang, and G. F. Tseng, "The immediate large-scale dendritic plasticity of cortical pyramidal neurons subjected to acute epidural compression," Neuroscience, vol. 167, no. 2, pp. 414-427, 2010.

[17] M. Dziembowska and J. Wlodarczyk, "MMP9: a novel function in synaptic plasticity," The International Journal of Biochemistry \& Cell Biology, vol. 44, no. 5, pp. 709-713, 2012.

[18] H. Frankowski, Y. H. Gu, J. H. Heo, R. Milner, and G. J. Del Zoppo, "Use of gel zymography to examine matrix metalloproteinase (gelatinase) expression in brain tissue or in primary glial cultures," in Astrocytes, vol. 814 of Methods in Molecular Biology, pp. 221-233, 2012.

[19] T. Klein and R. Bischoff, "Physiology and pathophysiology of matrix metalloproteases," Amino Acids, vol. 41, no. 2, pp. 271290, 2011.

[20] P. E. Van den Steen, B. Dubois, I. Nelissen, P. M. Rudd, R. A. Dwek, and G. Opdenakker, "Biochemistry and molecular biology of gelatinase B or matrix metalloproteinase-9 (MMP-9)," Critical Reviews in Biochemistry and Molecular Biology, vol. 37, no. 6, pp. 375-536, 2002.

[21] J. Vandooren, J. van Damme, and G. Opdenakker, “On the structure and functions of gelatinase $\mathrm{B} /$ matrix metallo proteinase-9 in neuroinflammation," Progress in Brain Research, vol. 214, pp. 193-206, 2014.

[22] C. Bonnans, J. Chou, and Z. Werb, "Remodelling the extracellular matrix in development and disease," Nature Reviews Molecular Cell Biology, vol. 15, no. 12, pp. 786-801, 2014.

[23] I. M. Ethell and D. W. Ethell, "Matrix metalloproteinases in brain development and remodeling: synaptic functions and targets," Journal of Neuroscience, vol. 85, no. 13, pp. 28132823, 2007.

[24] M. Ferrer-Ferrer and A. Dityatev, "Shaping synapses by the neural extracellular matrix," Frontiers in Neuroanatomy, vol. 12, p. 40, 2018.

[25] J. D. Mott and Z. Werb, "Regulation of matrix biology by matrix metalloproteinases," Current Opinion in Cell Biology, vol. 16, no. 5, pp. 558-564, 2004.

[26] G. Opdenakker, "On the roles of extracellular matrix remodeling by gelatinase B," Verhandelingen - Koninklijke Academie 
Voor Geneeskunde Van Belgie, vol. 59, no. 6, pp. 489-514, 1997.

[27] L. L. Phillips, T. M. Reeves, J. L. Chan, and A. E. Doperalski, "Time dependent integration of matrix metalloproteinases and their targeted substrates directs axonal sprouting and synaptogenesis following central nervous system injury," Neural Regeneration Research, vol. 9, no. 4, pp. 362-376, 2014.

[28] B. Pijet, M. Stefaniuk, A. Kostrzewska-Ksiezyk, P. E. Tsilibary, A. Tzinia, and L. Kaczmarek, "Elevation of MMP-9 levels promotes epileptogenesis after traumatic brain injury," Molecular Neurobiology, vol. 55, no. 12, pp. 9294-9306, 2018.

[29] B. Vafadari, A. Salamian, and L. Kaczmarek, "MMP-9 in translation: from molecule to brain physiology, pathology, and therapy," Journal of Neurochemistry, vol. 139, no. 2, pp. 91114, 2016.

[30] D. Neuhofer and P. Kalivas, "Metaplasticity at the addicted tetrapartite synapse: a common denominator of drug induced adaptations and potential treatment target for addiction," Neurobiology of Learning and Memory, vol. 154, pp. 97-111, 2018.

[31] P. Penzes, M. E. Cahill, K. A. Jones, J. E. VanLeeuwen, and K. M. Woolfrey, "Dendritic spine pathology in neuropsychiatric disorders," Nature Neuroscience, vol. 14, no. 3, pp. 285-293, 2011.

[32] A. Pitkänen, X. E. Ndode-Ekane, K. Łukasiuk et al., "Neural ECM and epilepsy," Progress in Brain Research, vol. 214, pp. 229-262, 2014.

[33] S. M. Reinhard, K. Razak, and I. M. Ethell, "A delicate balance: role of MMP-9 in brain development and pathophysiology of neurodevelopmental disorders," Frontiers in Cellular Neuroscience, vol. 9, p. 280, 2015.

[34] K. Lepeta and L. Kaczmarek, "Matrix metalloproteinase-9 as a novel player in synaptic plasticity and schizophrenia," Schizophrenia Bulletin, vol. 41, no. 5, pp. 1003-1009, 2015.

[35] M. Verslegers, K. Lemmens, I. Van Hove, and L. Moons, "Matrix metalloproteinase- 2 and -9 as promising benefactors in development, plasticity and repair of the nervous system," Progress In Neurobiology, vol. 105, pp. 60-78, 2013.

[36] M. Magnowska, T. Gorkiewicz, A. Suska et al., "Transient ECM protease activity promotes synaptic plasticity," Scientific Reports, vol. 6, no. 1, article 27757, 2016.

[37] P. Michaluk, M. Wawrzyniak, P. Alot et al., "Influence of matrix metalloproteinase MMP-9 on dendritic spine morphology," Journal Of Cell Science, vol. 124, no. 19, pp. 3369-3380, 2011.

[38] Z. Szepesi, E. Hosy, B. Ruszczycki et al., "Synaptically released matrix metalloproteinase activity in control of structural plasticity and the cell surface distribution of GluA1-AMPA receptors," PLoS One, vol. 9, no. 5, article e98274, 2014.

[39] L. Tian, M. Stefanidakis, L. Ning et al., "Activation of NMDA receptors promotes dendritic spine development through MMP-mediated ICAM-5 cleavage," Journal Of Cell Biology, vol. 178, no. 4, pp. 687-700, 2007.

[40] X. Wang, J. Jung, M. Asahi et al., "Effects of matrix metallo proteinase-9 gene knock-out on morphological and motor outcomes after traumatic brain injury," The Journal of Neuroscience, vol. 20, no. 18, pp. 7037-7042, 2000.

[41] X. Wang, O. Bozdagi, J. S. Nikitczuk, Z. W. Zhai, Q. Zhou, and G. W. Huntley, "Extracellular proteolysis by matrix metallo proteinase-9 drives dendritic spine enlargement and longterm potentiation coordinately," Proceedings of the National
Academy of Sciences of the United States of America, vol. 105, no. 49, pp. 19520-19525, 2008.

[42] T. Hayashi, Y. Kaneko, S. Yu et al., "Quantitative analyses of matrix metalloproteinase activity after traumatic brain injury in adult rats," Brain research, vol. 1280, pp. 172177, 2009.

[43] J. S. Truettner, O. F. Alonso, and W. D. Dietrich, "Influence of therapeutic hypothermia on matrix metalloproteinase activity after traumatic brain injury in rats," Journal of Cerebral Blood Flow \& Metabolism, vol. 25, no. 11, pp. 1505-1516, 2005.

[44] E. Suehiro, H. Fujisawa, T. Akimura et al., "Increased matrix metalloproteinase-9 in blood in association with activation of interleukin-6 after traumatic brain injury: influence of hypothermic therapy," Journal of Neurotrauma, vol. 21, no. 12, pp. 1706-1711, 2004.

[45] D. Vajtr, O. Benada, J. Kukacka et al., "Correlation of ultrastructural changes of endothelial cells and astrocytes occurring during blood brain barrier damage after traumatic brain injury with biochemical markers of BBB leakage and inflammatory response," Physiological Research, vol. 58, no. 2, pp. 263-268, 2009.

[46] T. Bolkvadze and A. Pitkänen, "Development of posttraumatic epilepsy after controlled cortical impact and lateral fluid-percussion-induced brain injury in the mouse," Journal of Neurotrauma, vol. 29, no. 5, pp. 789-812, 2012.

[47] T. H. Vu, J. M. Shipley, G. Bergers et al., "MMP-9/gelatinase B is a key regulator of growth plate angiogenesis and apoptosis of hypertrophic chondrocytes," Cell, vol. 93, no. 3, pp. 411-422, 1998.

[48] D. H. Smith, H. D. Soares, J. S. Pierce et al., "A model of parasagittal controlled cortical impact in the mouse: cognitive and histopathologic effects," Journal of Neurotrauma, vol. 12, no. 2, pp. 169-178, 1995.

[49] B. Ruszczycki, Z. Szepesi, G. M. Wilczynski et al., "Sampling issues in quantitative analysis of dendritic spines morphology," BMC Bioinformatics, vol. 13, no. 1, p. 213, 2012.

[50] H. Kasai, M. Fukuda, S. Watanabe, A. Hayashi-Takagi, and J. Noguchi, "Structural dynamics of dendritic spines in memory and cognition," Trends in Neurosciences, vol. 33, no. 3, pp. 121-129, 2010.

[51] M. Miller, "Maturation of rat visual cortex. III. Postnatal morphogenesis and synaptogenesis of local circuit neurons," Brain Research, vol. 390, no. 2, pp. 271-285, 1986.

[52] J. D. Jontes and S. J. Smith, "Filopodia, spines, and the generation of synaptic diversity," Neuron, vol. 27, no. 1, pp. 11-14, 2000.

[53] E. A. Nimchinsky, B. L. Sabatini, and K. Svoboda, "Structure and function of dendritic spines," Annual Review of Physiology, vol. 64, no. 1, pp. 313-353, 2002.

[54] R. Yuste, "Dendritic spines and distributed circuits," Neuron, vol. 71, no. 5, pp. 772-781, 2011.

[55] M. Wosiski-Kuhn and A. M. Stranahan, "Transient increases in dendritic spine density contribute to dentate gyrus longterm potentiation," Synapse, vol. 66, no. 7, pp. 661-664, 2012.

[56] J. Jarero-Basulto, Y. Gasca-Martínez, M. Rivera-Cervantes, M. Ureña-Guerrero, A. Feria-Velasco, and C. Beas-Zarate, "Interactions between epilepsy and plasticity," Pharmaceuticals, vol. 11, no. 1, p. 17, 2018.

[57] T. P. Sutula and F. E. Dudek, "Unmasking recurrent excitation generated by mossy fiber sprouting in the epileptic dentate gyrus: an emergent property of a complex system," Progress In Brain Research, vol. 163, pp. 541-563, 2007. 
[58] O. Hadass, B. N. Tomlinson, M. Gooyit et al., "Selective inhibition of matrix metalloproteinase- 9 attenuates secondary damage resulting from severe traumatic brain injury," PLoS One, vol. 8, no. 10, article e76904, 2013.

[59] M. Grossetete, J. Phelps, L. Arko, H. Yonas, and G. A. Rosenberg, "Elevation of matrix metalloproteinases 3 and 9 in cerebrospinal fluid and blood in patients with severe traumatic brain injury," Neurosurgery, vol. 65, no. 4, pp. 702-708, 2009.

[60] A. Szklarczyk, J. Lapinska, M. Rylski, R. D. G. McKay, and L. Kaczmarek, "Matrix metalloproteinase-9 undergoes expression and activation during dendritic remodeling in adult hippocampus," The Journal of Neuroscience, vol. 22, no. 3, pp. 920-930, 2002.

[61] G. M. Wilczynski, F. A. Konopacki, E. Wilczek et al., "Important role of matrix metalloproteinase 9 in epileptogenesis," Journal of Cell Biology, vol. 180, no. 5, pp. 1021-1035, 2008.

[62] A. Nagaoka, H. Takehara, A. Hayashi-Takagi et al., "Abnormal intrinsic dynamics of dendritic spines in a fragile $\mathrm{X}$ syndrome mouse model in vivo," Scientific Reports, vol. 6, no. 1, article 26651, 2016.

[63] A. Dityatev, M. Schachner, and P. Sonderegger, "The dual role of the extracellular matrix in synaptic plasticity and homeostasis," Nature Reviews Neuroscience, vol. 11, no. 11, pp. 735-746, 2010.

[64] G. W. Huntley, "Synaptic circuit remodelling by matrix metalloproteinases in health and disease," Nature Reviews Neuroscience, vol. 13, no. 11, pp. 743-757, 2012. 


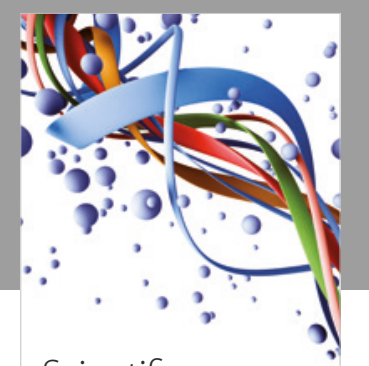

Scientifica
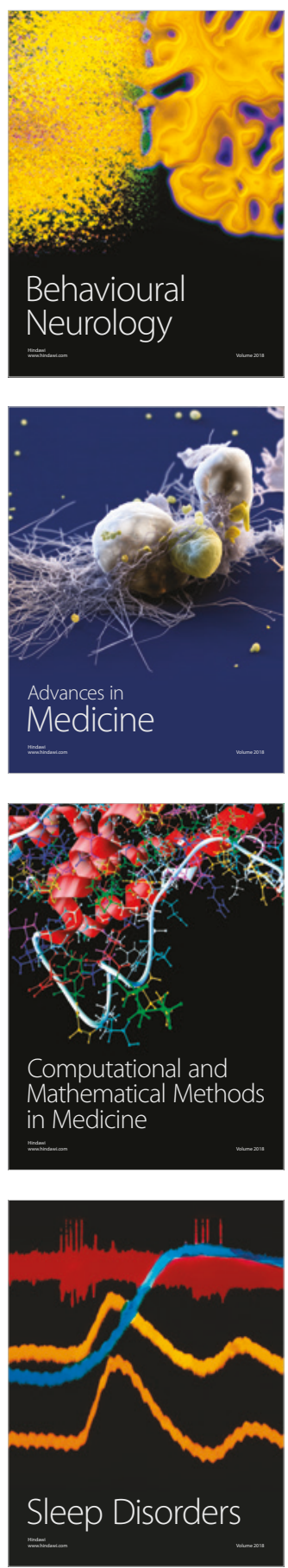

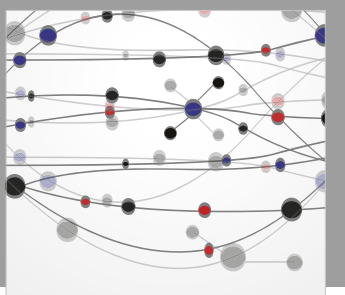

The Scientific World Journal

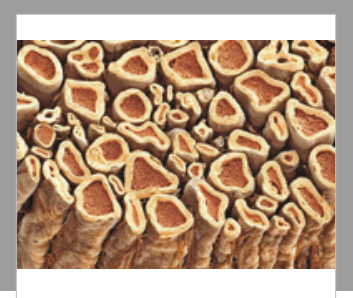

Case Reports in

Neurological Medicine

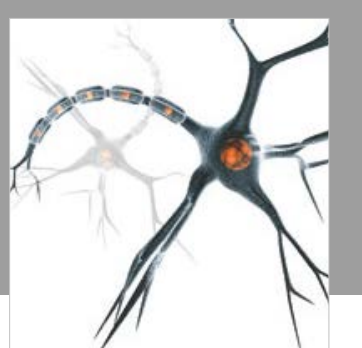

Neural Plasticity

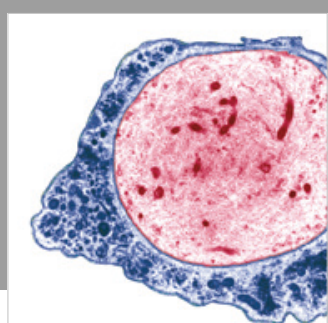

Multiple Sclerosis

International

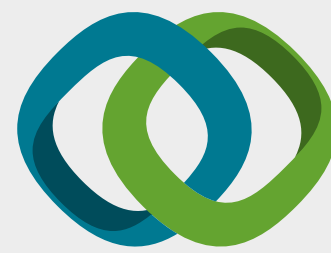

Hindawi

Submit your manuscripts at

www.hindawi.com
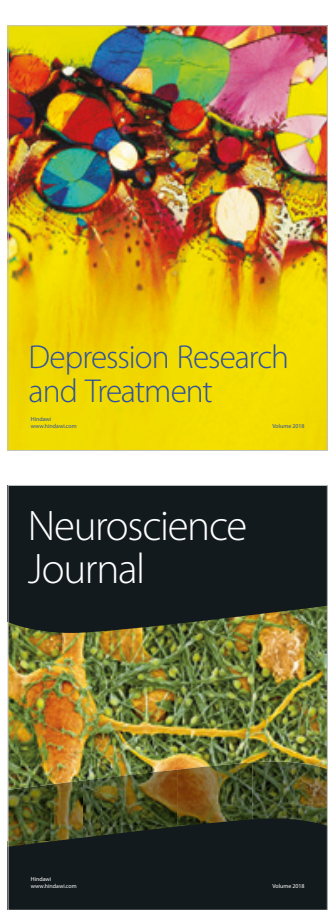

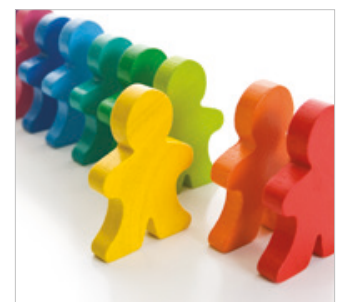

Autism

Research and Treatment
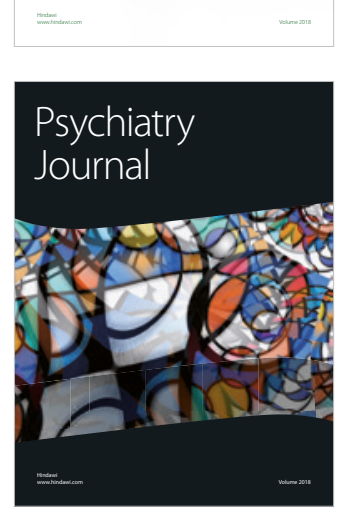
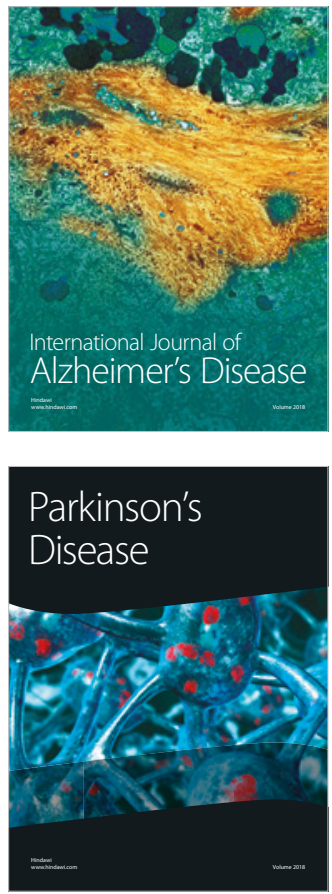
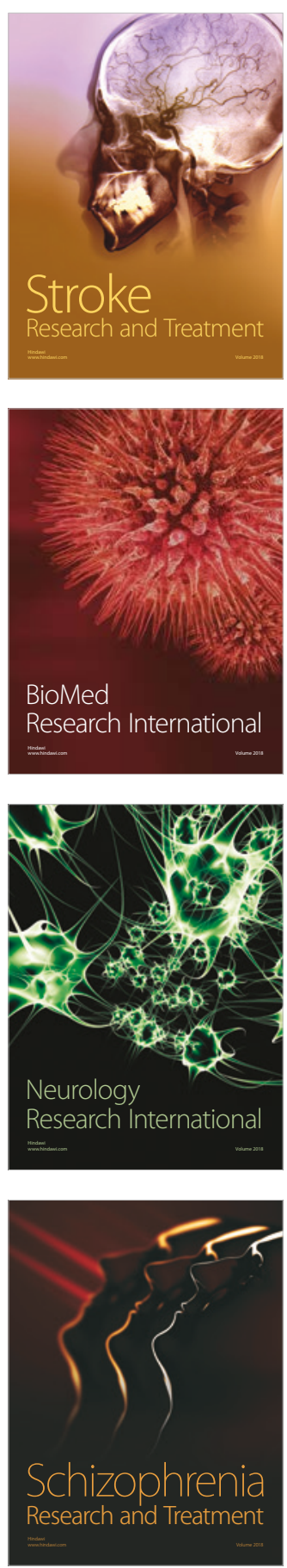